\title{
1-port SOL Calibration Method and the design of calibration kits of Double Waveguide
}

\author{
Sen Wang ${ }^{1,}$, $\mathrm{Hao} \mathrm{Li}^{2, \mathrm{~b}}$ and Shuo $\mathrm{Cui}^{3, \mathrm{c}}$ \\ ${ }^{1}$ No.1, Nan Da Hong Men Road, Fengtai District, Beijing, China \\ ${ }^{2}$ No.1, Nan Da Hong Men Road, Fengtai District, Beijing, China \\ ${ }^{3}$ No.2006, Xiyuan Ave, West Hi-Tech Zone, Chengdu,.China \\ a15908109077@163.com, b952077096@qq.com, c cshuofred@foxmail.com
}

Keywords: SOL calibration method, double waveguide, calibration kits, microwave testing.

Abstract. The calibration of vector network analyzer can reduce errors in the test system, which will significantly improves the accuracy of measurement. A short-ended double waveguide in this paper is a 1-port network used for measurement of microwave materials. SOL calibration method is very important to 1-port network and double waveguide has its unique application in the measurement of microwave materials. Based on the analysis of 1-port network's error sources, error models were established and corrected. Then the design of calibration kits of double waveguide was introduced. The test system was built to test the calibration efficiency, and the test result shows the calibration kits played a quite good effect in the measurement.

\section{Introduction}

Double waveguide is a kind of parallel transmission structure, so that its special structure contributes to the important application and unique advantages in the measurement of microwave materials. As shown in Fig.1, the sample and reference sample could be tested at the same time, which makes it easier to analyze the test result. However, double waveguide is a kind of device with non-standard flanges, so that calibration kits need be designed on our own.

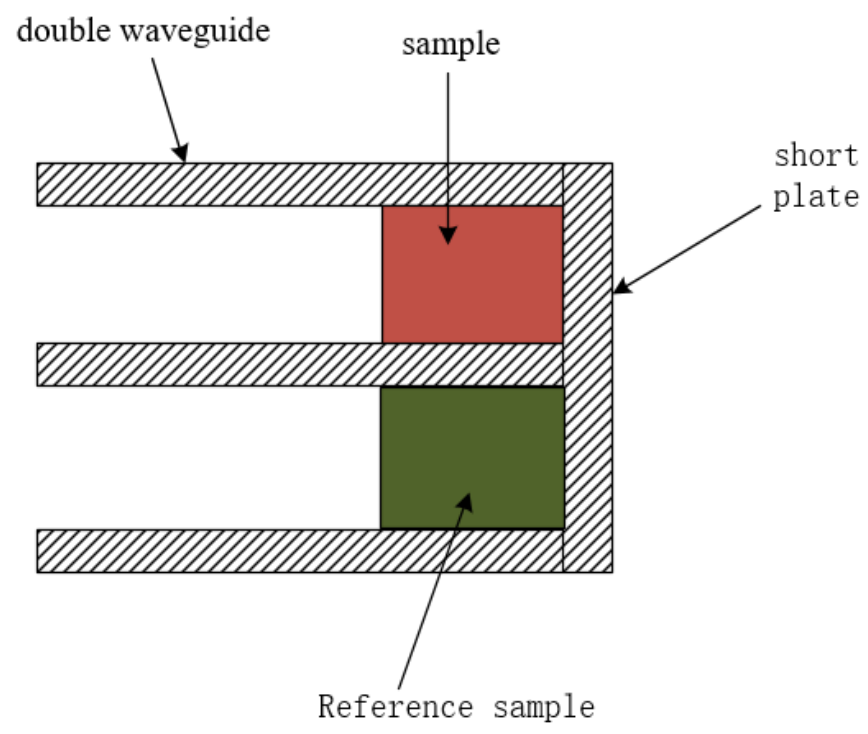

Fig. 1. Sketch of double waveguide.

At present, the most commonly used calibration methods of network analyzer include SOLT (Short-Open-Load-Thru) and TRL (Thru-Reflect-Line). Both of them are used for 2-port network. As to 1-port network, SOL (Short-Open-Load) is the most popular choice. Based on the analysis of the error source, the error model was built and the formula of error correction was deduced. In this paper, the design method of the calibration kits is introduced, and the calibration result is provided. The result shows that the calibration kits achieve the expected effect and can meet the test requirement of the double waveguide. 


\section{Analysis of Error Source and Error Model}

For a 1-port network, the parameters only include reflection coefficient and there is no transmission parameter. The system itself contains a lot of passive and active devices, which are not ideal and will bring lots of errors. The error source of 1-port network mainly include source mismatch error $\mathrm{E}_{\mathrm{S}}$ frequency response tracking error $E_{R T}$ and direction error $E_{D}[1-3]$.

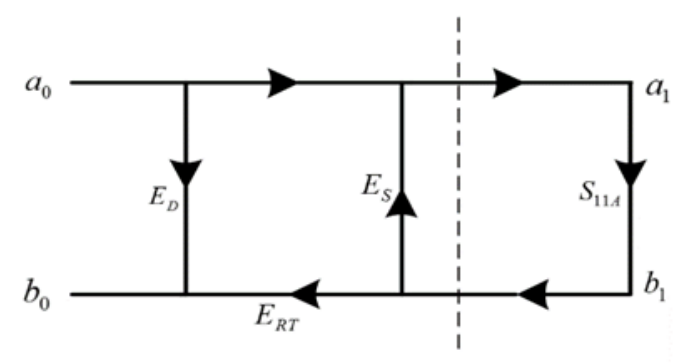

Fig. 2. System's signal flow graph.

1-port network's signal flow graph is shown in Fig.2. $S_{11 A}$ is the useful signal that goes through DUT and then is reflected and $S_{11 M}$ is the actual measured value. When $S_{11 \mathrm{~A}}$ is $S_{11 M S}=-1, S_{11 M O}=1$ or $S_{11 M L}=0$, the corresponding terminal state is short, open, load [4].

According to the Mason decomposition rule of the signal flow graph, the error model of 1-port network can be simplified as shown in Fig.3.

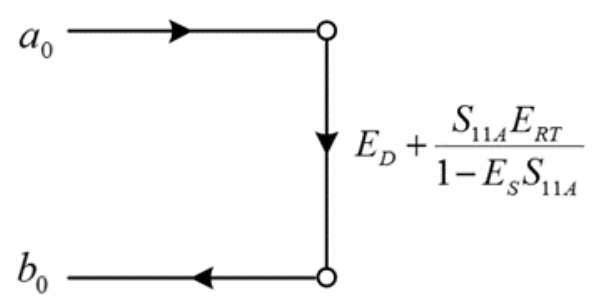

Fig. 3. Simplified signal flow graph

Then as $S_{11 M}$ is shown in Fig. $3, S_{11 A}$ can be deduced.

$$
S_{11 A}=\frac{S_{11 M}+E_{D}}{E_{R T}+E_{S}\left(S_{11 M}+E_{D}\right)}
$$

According to [4], we can know if we replace the DUT on the test port by calibration kits-short, open and load, $E_{S}, E_{R T}$ and $E_{D}$ can be deduced.

$$
\begin{aligned}
& E_{D}=S_{11 M L} \\
& E_{S}=\frac{S_{11 M S}+S_{11 M O}-2 S_{11 M L}}{S_{11 M O}-S_{11 M S}} \\
& E_{R T}=\frac{2\left(S_{11 M L}-S_{11 M S}\right)\left(S_{11 M O}-S_{11 M L}\right)}{S_{11 M O}-S_{11 M S}}
\end{aligned}
$$


Now we have value of $E_{S}, E_{R T}$ and $E_{D}$, and then bring them back into Equations.1.

$$
S_{11 A}=\frac{\left(S_{11 M}+S_{11 M L}\right)\left(S_{11 M O}-S_{11 M S}\right)}{2\left(S_{11 M L}-S_{11 M S}\right)\left(S_{11 M O}-S_{11 M L}\right)+\left(S_{11 M S}+S_{11 M O}-2 S_{11 M L}\right)\left(S_{11 M}+S_{11 M L}\right)}
$$

The calibration method is used to measure three errors, and then the DUT is tested. The three errors will be deducted from the test result of DUT. This process is called 1-port network error correction.

\section{Design of Calibration Kits}

A kind of X-band double rectangular waveguide is used in the test system, and it is a non-standard waveguide. So the calibration kits need be designed, and SOL calibration method itself also has the corresponding request.

1. SHORT: the short circuit of waveguide is usually produced by a short-circuit board. The amplitude of reflection coefficient should be close to 1 , and the phase should be close to $\pm 180^{\circ}$.

2. OPEN: when a transmission line is open, the edge will bring out parasitic capacitance, which will affect the results of the test. In order to eliminate the influence of parasitic capacitance, a $\lambda / 4$ waveguide and a short-circuit board are used to realize the open circuit.

3. LOAD: a taper-type matched load was placed in a short-ended waveguide to achieve the matching of the waveguide.

As shown in Fig.4, all the calibration kits are provided with a positioning hole the same as the waveguide to ensure precise docking. And they were all used brass as the processing material. The surface of the short circuit board is polished. Matched load waveguide is made up of two halves of waveguide machined by milling cutter to ensure the waveguide wall flat and smooth, meanwhile current of the waveguide surface won't be affected. Finally, plate gold on all the kits.

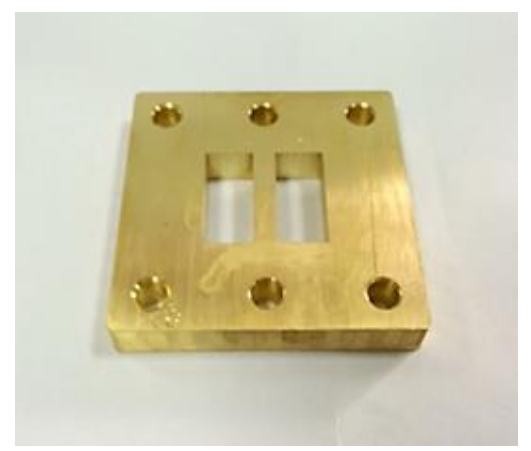

(a)

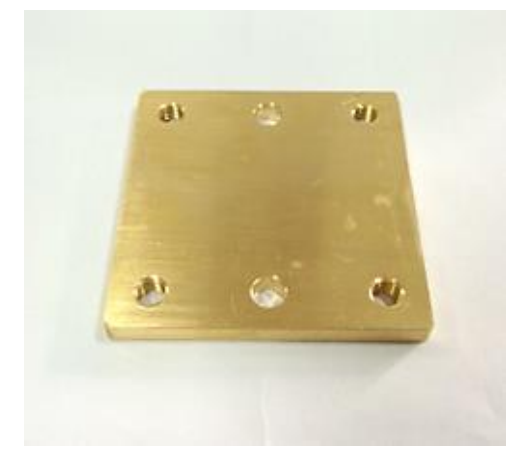

(b)

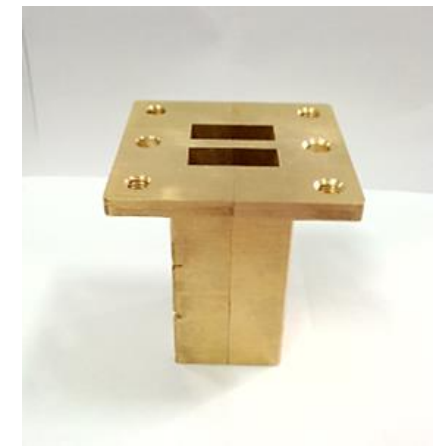

(c)

Fig. 4. Calibration kits: (a) $\lambda / 4$ waveguide (b) Short (c) Load.

\section{Result and Analysis}

Build a test system as Fig.5. Then choose 1-Port SOL calibration on the vector network analyzer and follow the prompts to complete the calibration process. 


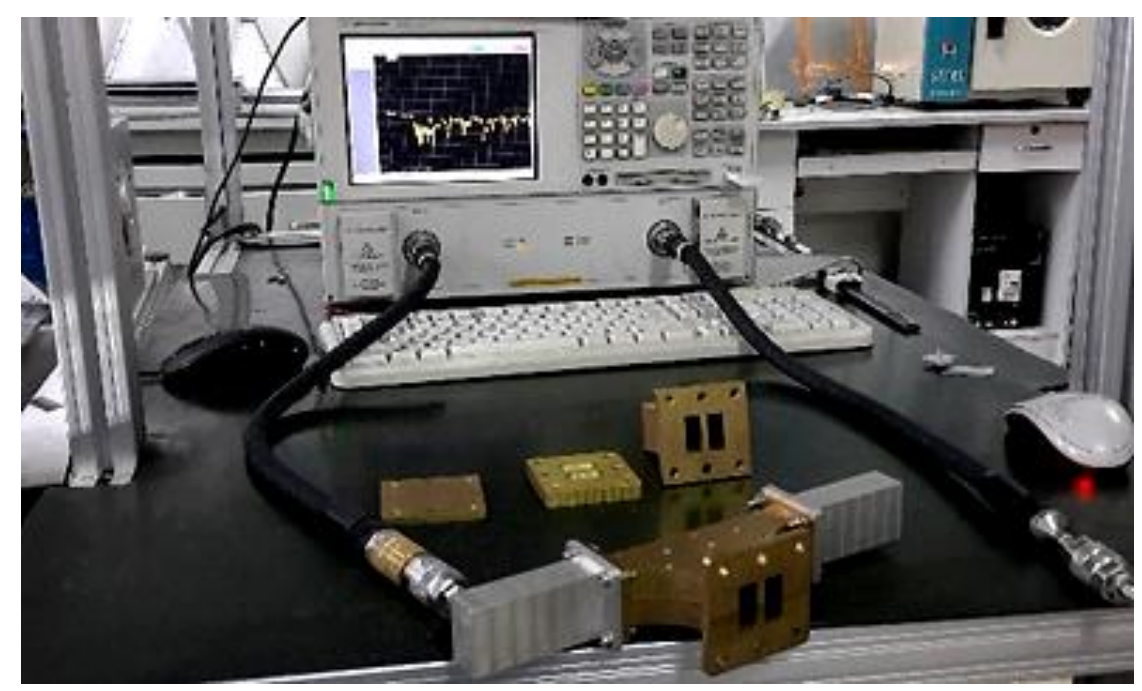

Fig.5. Testing system.

When the calibration process was done, the kits were connected to the waveguide again to test their calibration efficiency.

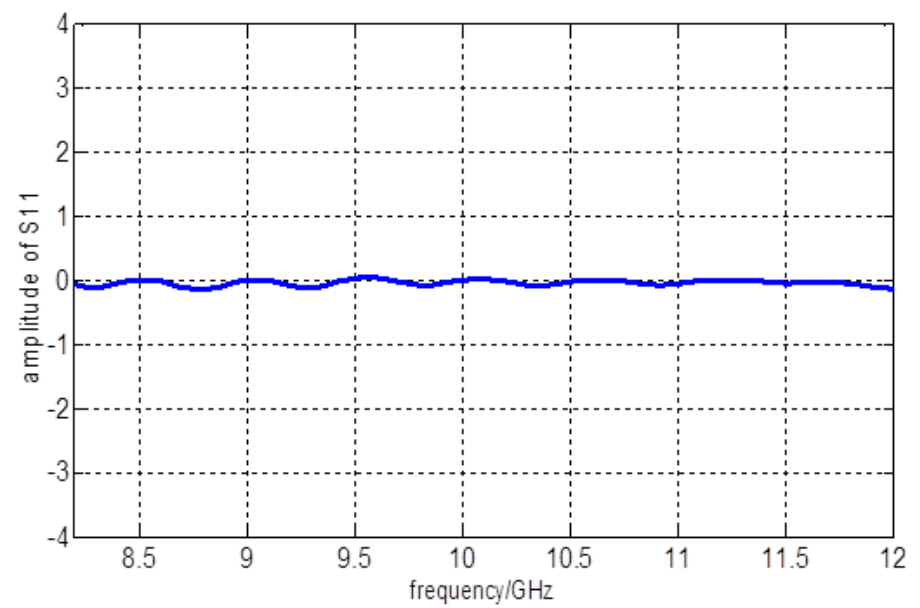

(a)

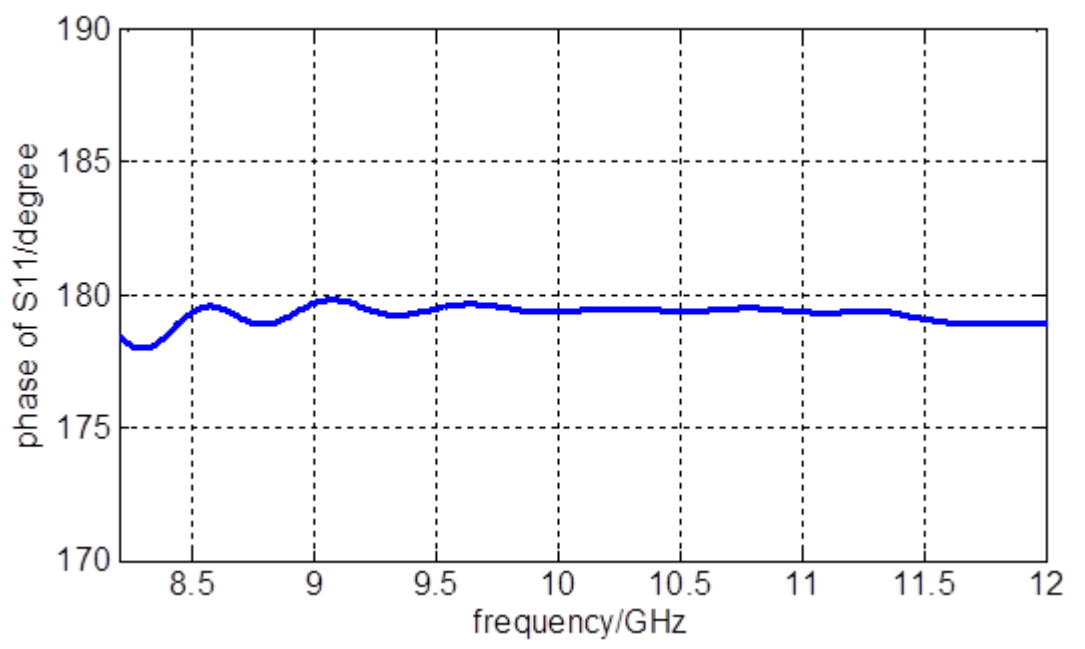

(b)

Fig.6. S 11 of Short: (a) Amplitude (b) Phase

As Fig. 6 shows, the amplitude of $S_{11}$ is very close to $0 \mathrm{~dB}$ and the phase is nearly $180^{\circ}$. Obviously, Short meets the demand of design. 


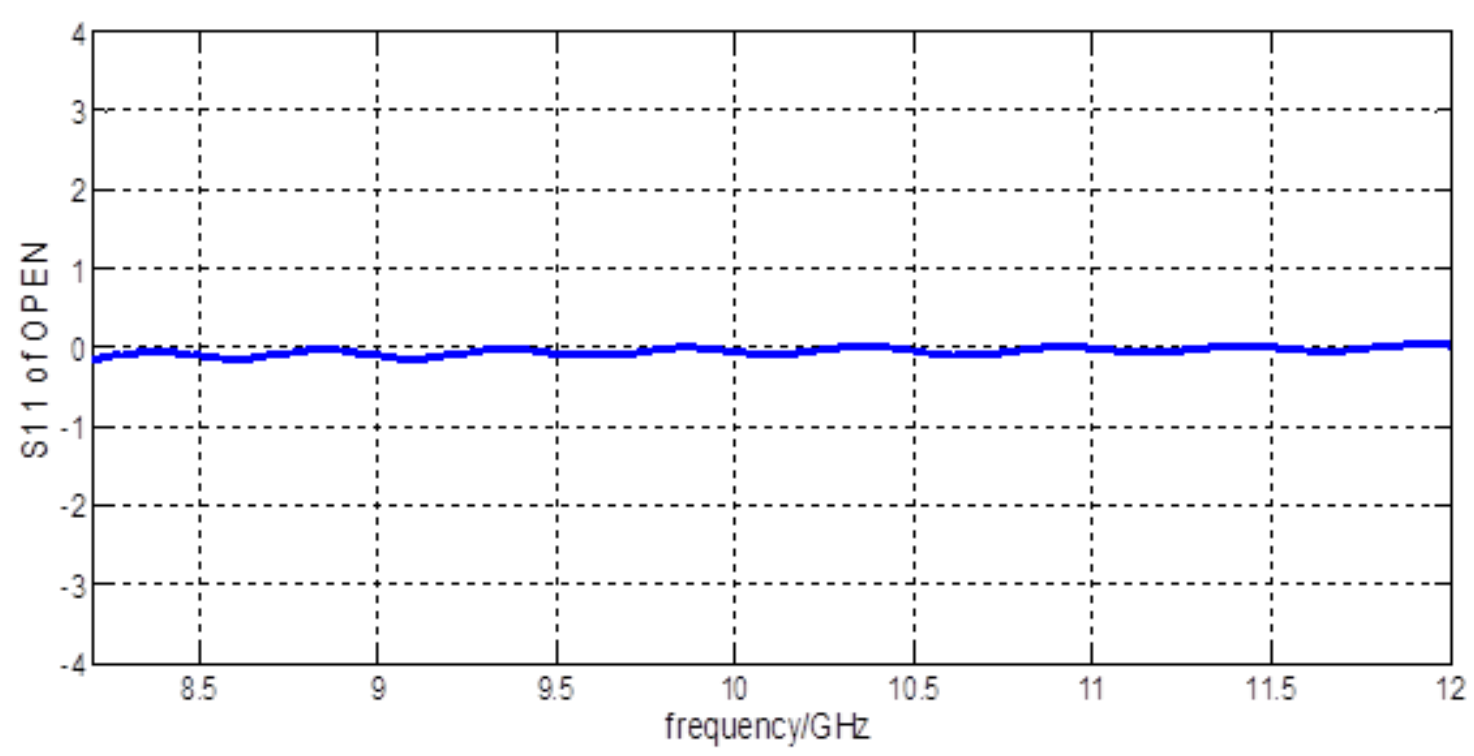

Fig.7. Amplitude of $\mathrm{S}_{11}$ when Open was connected

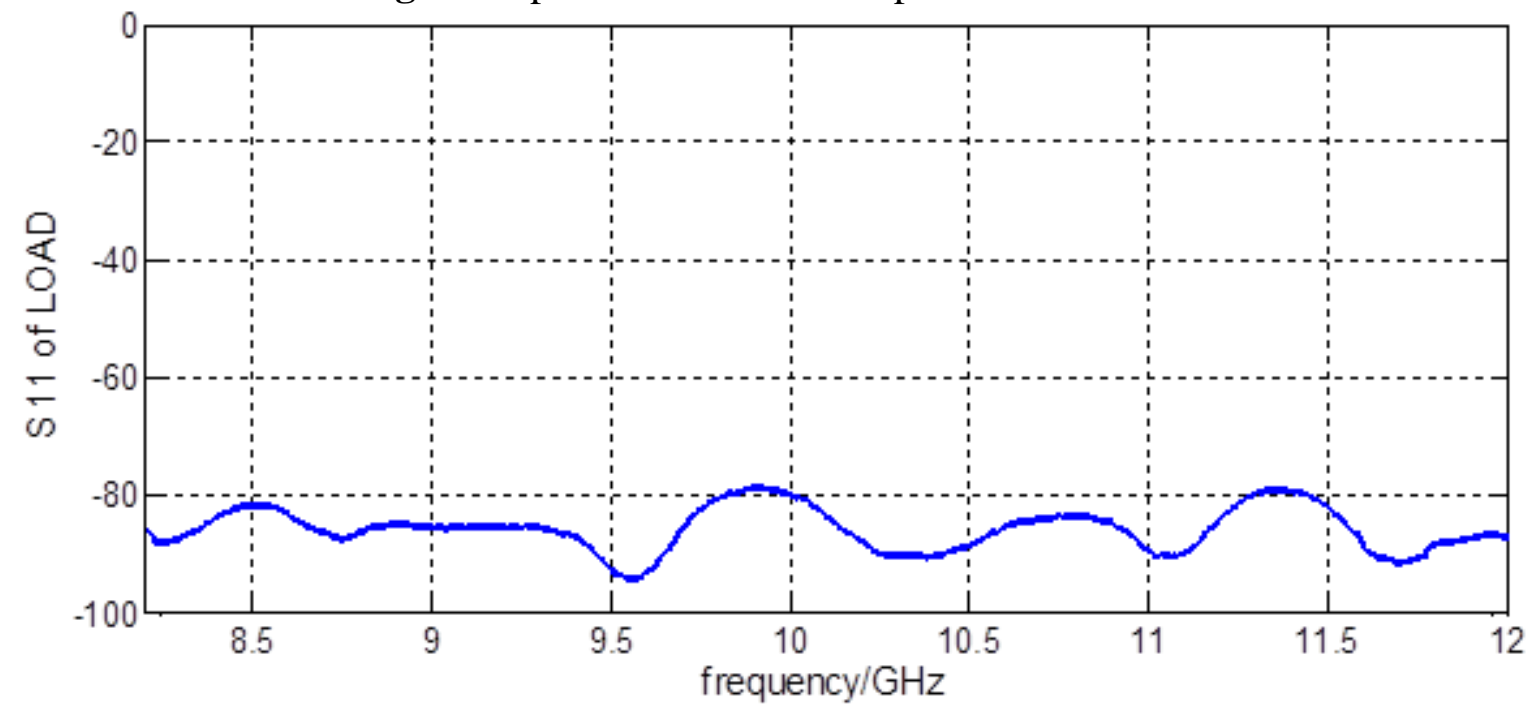

Fig.8. Amplitude of S11 when Load was connected

Fig. 7 shows that amplitude of Open is nearly $0 \mathrm{~dB}$, and in Fig. 8 we can see that amplitude of Load is less than $-70 \mathrm{~dB}$ in X-band. So Open and Load also performed well in the calibration process.

\section{Conclusions}

In this paper, firstly double waveguide and its application in the measurement of microwave materials were introduced. Then we analyzed 1-port network's error sources and SOL calibration method. The design of the calibration kits complies with corresponding rules to ensure the calibration efficiency and were machined in high precision.

The test results show that the calibration kits accord with the design requirements and have played a good effect in the experiment. We can say that the calibration kits are very effective in measurement and will significantly improve the test precision of microwave materials.

\section{References}

[1] U.Stumper, A.J.Slobodnik, G.A.Roberts. Influence of TMSO calibration standards uncertainties on VNA s-parameter measurements [J]. IEEE Transactions on Instrumentation and Measurement, (2003), 52(2): 311-345 
[2] Xiaoming Tang. Single-reflection method for the temperature-dependence measurement of complex permittivity of planar dielectric materials [D]. Chengdu: University of Electronic Science and Technology of China (2011)

[3] Ling Tong. Covariance-matrix-based uncertainty propagation analysis for s-parameter measurements and application [D]. Harbin: Harbin Institute of Technology (2012), in Chinese

[4] S. Padmanabhan, P. Kirby, J. Daniel, et al. Accurate Broadband On-Wafer SOLT Calibrations with Complex Load and Thru Models [C]. 62nd ARFTG Conference Digest, (2003):5-10 